\title{
Detectability of surface-laid landmines with a polarimetric IR sensor
}

\author{
Frank Cremer $^{a b c}$, Wim de Jong ${ }^{a}$, Klamer Schutte ${ }^{a}$, Wen-Jiao Liao ${ }^{d}$ and Brian A. Baertlein ${ }^{d}$ \\ ${ }^{a}$ TNO Physics and Electronics Laboratory, P.O. Box 96864, 2509 JG, The Hague, The Netherlands \\ ${ }^{b}$ Pattern Recognition Group, Delft University of Technology, Delft, The Netherlands \\ ${ }^{c}$ Section of Applied Geophysics, Delft University of Technology, Delft, The Netherlands \\ ${ }^{d}$ Ohio State University ElectroScience Laboratory, Columbus, OH, USA
}

\begin{abstract}
Polarimetric scattering models are developed to predict the detectability of surface-laid landmines. A specular polarimetric model works well only under the condition that there is either no sunlight or the sun is not close to the specular reflection direction. Moreover, this model does not give insight why certain man-made objects like landmines give a higher polarimetric signature than natural background. By introducing a polarimetric bidirectional reflectance distribution function (BRDF) the specular model is extended. This new model gives a better prediction of the polarimetric signature and gives a close match to the measurements of landmines with different casings as well as the sand background. The model parameters indicate that the landmines have a lower surface roughness and a higher refractive index, which is the reason why these objects are detectable from the background based on their polarimetric signature.
\end{abstract}

Keywords: polarimetric infrared, modelling, BRDF, landmine detection

\section{INTRODUCTION}

One of the sensors that is considered for a multi-sensor landmine detection system is the infrared (IR) camera. ${ }^{1}$ This infrared camera can be enhanced by means of a polarisation filter. ${ }^{2}$ By using this filter not only differences in thermal properties (for buried and surface-laid landmines) can be observed but also surface properties (for surface-laid landmines).

The surface properties are the refractive index and the surface roughness. For a smooth surface, the refractive index determines how much of the incoming radiation is reflected toward the polarimetric sensor in the specular direction. For a rough surface, both the refractive index and the surface texture or roughness affect the distribution of reflections in all possible directions.

In a specular model described previously by the authors, the simplification is made that the radiation coming from the specular direction is the only contributing factor. This simplification seems to work well for indoor measurements ${ }^{2}$ and reasonable well for one outdoor experiment. ${ }^{3}$ However, there are two problems with this approach. First, it does not explain why there is polarimetric radiation reflected (and emitted) from landmines and much less from the background. Second, there are situations as shown in this paper where the specular model does not work very well.

The situations for which the specular model does not work are largely determined by the position and visibility of the sun. When the sun is shining and it is close enough to the specular path, then the solar radiation starts to overpower both the emission from the landmine and the reflection from the sky. To include this sun effect in our model we extended it with a polarimetric Bidirectional Reflectance Distribution Function (BRDF) model. This model was already included in a polarimetric image simulator. ${ }^{4}$ This image simulator produces images that are quite similar to the measured images using estimates of the refractive indices and surface roughnesses.

The model parameters are estimated from measurements. The error in the fit of the measurement results gives an indication of whether or not this model is applicable. The variation of the parameters over landmines of one type gives an indication of how accurate the parameters are estimated. Furthermore the parameters from the background are estimated.

Further author information: (Send correspondence to Frank Cremer)

F.C.: E-mail: FCremer@etro.vub.ac.be Phone: +32 (2) 6292787 Fax: +32 (2) 6292883

B.A.B.: E-mail: baertlein.1@osu.edu, Telephone: +1 614292 0076, The Ohio State University, ElectroScience Laboratory, 1320 Kinnear Road, Columbus, OH, 43212 USA 
The estimated model parameters from the landmines and the background give insight in the detectability of landmines under various conditions.

The two polarimetric models are introduced in Section 2. The measurement setup and instrumentation is discussed in Section 3. This is followed by a series of measurements in Section 4. The combined model is validated and the model parameters are given in Section 5. Finally, this paper ends with a discussion and conclusions in Section 6.

\section{POLARIMETRIC IR MODELLING}

Two different approaches have been taken to model the polarimetric IR radiation that is reflected and emitted by a surface. The first approach is the most basic approach and models only emission and specular reflection and neglects diffuse reflection, see Sec. 2.1. This model uses the sky radiance and the temperature of the landmine as input and predicts the resulting Stokes vector. This model has been validated previously ${ }^{3}$ and seems to perform well if the amount of direct sun light is limited.

In another approach a surface is modelled as a scatterer by means of a polarimetric bidirectional reflectance distribution function (BRDF), see Sec. 2.2. This approach has previously been incorporated in a polarimetric image simulator. ${ }^{4}$ Comparison between model images and measured images has shown reasonable agreement. However, for this model, the refractive index and the surface roughness must be specified. For most landmine materials these parameters are unknown.

The specular model works well for smooth surfaces and for rough surfaces when no localised sources like the sun are present. A more detailed model is required when these conditions are violated.

\subsection{Specular model}

In a previous publication it was shown that when the sun is not in the specular direction, the Stokes parameters $\left(I_{m}\right.$ and $Q_{m}$ ) for a horizontally oriented landmine with a smooth surface, depend on the sky radiance in the specular path ${ }^{2}$ :

$$
\begin{aligned}
I_{m} & =\frac{1}{2}\left[\rho_{s}\left(\theta_{o}\right)+\rho_{p}\left(\theta_{o}\right)\right]\left[I_{s k y}-I_{B B}\left(T_{m}\right)\right]+I_{B B}\left(T_{m}\right), \\
Q_{m} & =\frac{1}{2}\left[\rho_{s}\left(\theta_{o}\right)-\rho_{p}\left(\theta_{o}\right)\right]\left[I_{s k y}-I_{B B}\left(T_{m}\right)\right]
\end{aligned}
$$

with $\rho_{s}\left(\theta_{o}\right)$ and $\rho_{p}\left(\theta_{o}\right)$ the reflection coefficients of the landmine surface (which depend on the refractive index $n_{2}$ ), $T_{m}$ the blackbody surface temperature, $I_{s k y}$ the sky radiance and $\theta_{o}$ the observer angle (with the surface normal). In the specular model the observer angle is identical to the angle of incidence $\theta_{i}$. The third Stokes parameter $U_{m}$ is zero for this configuration. The blackbody radiance in IR is given by an integration of the Planck equation:

$$
I_{B B}(T)=\int_{\lambda_{1}}^{\lambda_{2}} \frac{2 h c^{2}}{\lambda^{5}} \frac{1}{e^{\frac{h c}{\lambda k T}}-1} d \lambda \quad\left[\mathrm{W} / \mathrm{m}^{2} / \mathrm{sr}\right],
$$

with $\lambda$ the wavelength, $\lambda_{1}$ to $\lambda_{2}$ the wavelength interval, $h$ Planck's constant, $k$ Boltzmann's constant and $c$ the speed of light.

When the land mine is not smooth or when the viewing geometry includes a direct solar reflection, the following more detailed analysis is required: The Stokes vector that describes the radiance produced by the mine in observer direction $\left(\theta_{o}, \phi_{o}\right)$ has contributions from emitted and reflected radiance. We can write

$$
S\left(\theta_{o}, \phi_{o}\right)=S_{r e f l}\left(\theta_{o}, \phi_{o}\right)+S_{e m}\left(\theta_{o}, \phi_{o}\right) .
$$

The emitted component $S_{e m}$ is approximated by the response of a smooth surface. We have

$$
S_{e m}=\left(\begin{array}{c}
1-\frac{1}{2}\left[\rho_{s}\left(\theta_{o}\right)+\rho_{p}\left(\theta_{o}\right)\right] \\
-\frac{1}{2}\left[\rho_{s}\left(\theta_{o}\right)-\rho_{p}\left(\theta_{o}\right)\right] \\
0 \\
0
\end{array}\right) I_{B B}\left(T_{m}\right)
$$


The reflected component is given by an integral over the upper hemisphere of the incident Stokes vector $S_{\text {inc }}$ times the Mueller matrix $M$ of the mine surface. We have

$$
S_{\text {refl }}\left(\theta_{o}, \phi_{o}\right)=\iint d \theta_{i} d \phi_{i} \sin \theta_{i} \cos \theta_{i} M\left(\theta_{o}, \phi_{o} \mid \theta_{i}, \phi_{i}\right) S_{i n c}\left(\theta_{i}, \phi_{i}\right) .
$$

The primary contributors to the incident Stokes vector $S_{i n c}$ are direct sunlight $S_{\text {sun }}$ and skylight $S_{s k y}$. Sunlight is a very localised source, and its angular dependence is well approximated by a Dirac delta function in solid angle at $\left(\theta_{\text {sun }}, \phi_{\text {sun }}\right)$. We have

$$
S_{\text {refl }}\left(\theta_{o}, \phi_{o}\right) \approx M\left(\theta_{o}, \phi_{o} \mid \theta_{\text {sun }}, \phi_{\text {sun }}\right) S_{\text {sun }}\left(\theta_{\text {sun }}, \phi_{\text {sun }}\right)+\iint d \theta_{i} d \phi_{i} \sin \theta_{i} \cos \theta_{i} M\left(\theta_{o}, \phi_{o} \mid \theta_{i}, \phi_{i}\right) S_{s k y}\left(\theta_{i}, \phi_{i}\right) .
$$

When the surface is relatively smooth and the specular and solar directions are far apart, the solar contribution can be neglected, as was done previously. ${ }^{2,3}$

Skylight is a distributed source, which necessitates an evaluation of the above integral. Prior work by the authors suggests that the value of the integral for an omni-directional source is well approximated by the value of the Mueller matrix for a smooth specular surface. We have

$$
S_{\text {refl }}\left(\theta_{o}, \phi_{o}\right) \approx M\left(\theta_{o}, \phi_{o} \mid \theta_{\text {sun }}, \phi_{\text {sun }}\right) S_{\text {sun }}\left(\theta_{\text {sun }}, \phi_{\text {sun }}\right)+M_{\text {spec }}\left(\theta_{o}, \phi_{o} \mid \theta_{i}, \phi_{i}\right) S_{\text {sky }}\left(\theta_{i}, \phi_{i}\right)
$$

in which $\left(\theta_{i}, \phi_{i}\right)$ is the incident angle required for specular reflection towards the observer.

Collecting the above results, we have

$$
S \approx S_{\text {em }}+M_{\text {spec }} S_{\text {sky }}+M S_{\text {sun }}
$$

We refer to this expression as the "combined model" in what follows. The specular reflection $M_{\text {spec }}$ in the above expression is given by:

$$
M_{\text {spec }}=\left(\begin{array}{cccc}
\frac{1}{2}\left[\rho_{s}\left(\theta_{i}\right)+\rho_{p}\left(\theta_{i}\right)\right] & m_{12} & m_{13} & m_{14} \\
\frac{1}{2}\left[\rho_{s}\left(\theta_{i}\right)-\rho_{p}\left(\theta_{i}\right)\right] & m_{22} & m_{23} & m_{24} \\
0 & m_{32} & m_{33} & m_{34} \\
0 & m_{42} & m_{43} & m_{44}
\end{array}\right),
$$

with $m_{i j}$ elements that have not been determined, but that are also not relevant for the following calculations, since the sky radiance is assumed to be not polarised. So the sky radiance is written as a Stokes vector $S_{s k y}$ :

$$
S_{s k y}=\left(\begin{array}{c}
I_{s k y} \\
0 \\
0 \\
0
\end{array}\right)
$$

with $I_{s k y}$ is the unpolarised sky radiance.

\subsection{BRDF model}

A variety of physically based, rough-surface BRDF models have been described in the literature including (but not limited to) the classical diffuse (Lambertian) model, the Beckmann-Spizzichino model ${ }^{5}$ (based on physical optics (PO)), the Torrance-Sparrow ${ }^{6}$ model (based on geometrical optics (GO)), and the semi-empirical Beard-Maxwell model. ${ }^{7}$ Of these, only the Beard-Maxwell and Beckmann models appear to have seen significant use for polarimetric sensors.

In this work we employ the Beckmann-Spizzichino model, which is particularly attractive for its rigorous development. We use a formulation described by Tsang et al. ${ }^{8}$ in which the PO integral is approximated by its stationary phase (geometrical optics) contribution. We include in the model both a multiplicative factor that accounts for self-shadowing in a manner described by Torrance and Sparrow and an additive term that accounts for diffuse subsurface scattering. In this 
work it is more convenient to work with the modified Stokes vector given by $S=\left[I_{h} I_{v} U V\right]^{T}$ in which $I_{v}$ and $I_{h}$ are the intensity polarised in the vertical and horizontal directions. The proposed Mueller matrix is

$$
\mathbf{M}\left(\hat{\mathbf{k}}_{s} \mid \hat{\mathbf{k}}_{i}\right)=\left[\mathbf{R}\left(\hat{\mathbf{k}}_{s} \mid \hat{\mathbf{k}}_{i}, \hat{\mathbf{n}}\right) G\left(\hat{\mathbf{k}}_{s} \mid \hat{\mathbf{k}}_{i}, \hat{\mathbf{n}}\right)+\mathbf{D}\right]\left[\begin{array}{cccc}
1 & 1 & 0 & 0 \\
1 & -1 & 0 & 0 \\
0 & 0 & 1 & 0 \\
0 & 0 & 0 & 1
\end{array}\right]
$$

in which $\hat{\mathbf{k}}_{s}$ and $\hat{\mathbf{k}}_{i}$ are unit vectors in the incident and scattered directions, and $\hat{\mathbf{n}}$ is the surface's unit normal. In addition, the component $R$ is the matrix for scattering by a rough surface derived by Tsang et al. ${ }^{8}$ It includes scalar factors that depend on the surface roughness and the source-target-viewer geometry as well as a matrix factor that embodies the polarisation dependence. Detailed descriptions of these components appear below.

As noted above, $\mathbf{R}$ is derived using the geometrical optics limit of the PO formulation. As a result, the PO formulation becomes very similar to the GO form (i.e., the Torrance-Sparrow model), which unfortunately includes a non-physical response at grazing angles. The factor $G$ is the self-obscuration or "geometric attenuation" function described previously in the Torrance and Sparrow model and given by

$$
G=\min \left\{1, \frac{2\left(\hat{\mathbf{n}}^{\prime} \cdot \hat{\mathbf{n}}\right)\left(\hat{\mathbf{k}}_{s} \cdot \hat{\mathbf{n}}\right)}{\left(\hat{\mathbf{n}}^{\prime} \cdot \hat{\mathbf{k}}_{s}\right)}, \frac{2\left(\hat{\mathbf{n}}^{\prime} \cdot \hat{\mathbf{n}}\right)\left(-\hat{\mathbf{k}}_{i} \cdot \hat{\mathbf{n}}\right)}{\left(\hat{\mathbf{n}}^{\prime} \cdot \hat{\mathbf{k}}_{s}\right)}\right\}
$$

This component provides a needed correction when either the source or viewing angles are near grazing. Finally, the component $\mathbf{D}$ accounts for diffuse scattering in the form

$$
\mathbf{D}=\frac{1}{2 \pi}\left[\begin{array}{cccc}
\rho_{d c} & \rho_{d x} & 1 & 1 \\
\rho_{d x} & \rho_{d c} & 1 & 1 \\
0 & 0 & 0 & 0 \\
0 & 0 & 0 & 0
\end{array}\right]
$$

in which $\rho_{d c}$ and $\rho_{d x}$ are diffuse albedos for co-polarised and cross-polarised scattering. Non-unit albedos could also be specified for the other polarisations, but at this time we have no data to guide their selection.

The PO formulation of Beckmann and Spizzichino is based on the assumption that the surface height distribution at any given point is a Gaussian random variable with zero mean and variance $\sigma^{2}$. The correlation $C$ between surface heights measured at any two points separated by a distance $\tau$ is also assumed to have the Gaussian form $C(\tau)=\exp \left(-\frac{\tau^{2}}{\ell^{2}}\right)$ in which $\ell$ is the distance between two points in the local plane. The surface height standard deviation $\sigma$ is required to be large comparing to the wavelength, and the correlation distance should be large enough so that no sharp edges are present on the surface. The ratio $s^{2}=2 \sigma^{2} / \ell^{2}$ is the mean-squared surface slope and is an important parameter in the analysis. A long derivation ${ }^{8}$ yields the following result for a surface element:

$$
\mathbf{R}\left(\hat{\mathbf{k}}_{s} \mid \hat{\mathbf{k}}_{i}\right)=\frac{1}{\cos \theta_{s}} \frac{\left|\overline{\mathbf{k}}_{1 d}\right|^{4}}{8 \pi s^{2}\left|\hat{\mathbf{k}}_{i} \times \hat{\mathbf{k}}_{s}\right|^{4}\left(\overline{\mathbf{k}}_{1 d} \cdot \hat{\mathbf{n}}\right)^{4}} \exp \left[-\frac{\left|\overline{\mathbf{k}}_{1 d t}\right|^{2}}{2 s^{2}\left(\overline{\mathbf{k}}_{1 d} \cdot \hat{\mathbf{n}}\right)^{2}}\right] \mathbf{C}
$$

in which $\overline{\mathbf{k}}_{1 d}=\hat{\mathbf{k}}_{i}-\hat{\mathbf{k}}_{s}, \overline{\mathbf{k}}_{1 d t}=\overline{\mathbf{k}}_{1 d}-\hat{\mathbf{n}}\left(\overline{\mathbf{k}}_{1 d} \cdot \hat{\mathbf{n}}\right)$ and

$$
\mathbf{C}=\left[\begin{array}{cccc}
<f_{h h} f_{h h}^{*}> & <f_{v h} f_{v h}^{*}> & R e<f_{h h} f_{v h}^{*}> & -I m<f_{h h} f_{v h}^{*}> \\
<f_{h v} f_{h v}^{*}> & <f_{v v} f_{v v}^{*}> & R e<f_{h v} f_{v v}^{*}> & -I m<f_{h v} f_{v v}^{*}> \\
2 R e<f_{h h} f_{h v}^{*}> & 2 R e<f_{v h} f_{v v}^{*}> & R e<f_{h h} f_{v v}^{*}+f_{v h} f_{h v}^{*}> & -I m<f_{h h} f_{v v}^{*}+f_{v h} f_{h v}^{*}> \\
2 I m<f_{h h} f_{h v}^{*}> & 2 I m<f_{v h} f_{v v}^{*}> & I m<f_{h h} f_{v v}^{*}+f_{v h} f_{h v}^{*}> & R e<f_{h h} f_{v v}^{*}+f_{v h} f_{h v}^{*}>
\end{array}\right]
$$

where $f_{h h}, f_{v v}, f_{h v}$, and $f_{v h}$ describe couplings among horizontal and vertical polarisations according to the sourceobserver geometry and the Fresnel reflection coefficients $R_{h}$ and $R_{v}$ (which depend on the refractive index $n_{1}$ ):

$$
\begin{aligned}
f_{h h} & =\left(\hat{\mathbf{v}}_{s} \cdot \hat{\mathbf{k}}_{i}\right)\left(\hat{\mathbf{v}}_{i} \cdot \hat{\mathbf{k}}_{s}\right) R_{h}+\left(\hat{\mathbf{h}}_{s} \cdot \hat{\mathbf{k}}_{i}\right)\left(\hat{\mathbf{h}}_{i} \cdot \hat{\mathbf{k}}_{s}\right) R_{v} \\
f_{v v} & =\left(\hat{\mathbf{h}}_{s} \cdot \hat{\mathbf{k}}_{i}\right)\left(\hat{\mathbf{h}}_{i} \cdot \hat{\mathbf{k}}_{s}\right) R_{h}+\left(\hat{\mathbf{v}}_{s} \cdot \hat{\mathbf{k}}_{i}\right)\left(\hat{\mathbf{v}}_{i} \cdot \hat{\mathbf{k}}_{s}\right) R_{v} \\
f_{h v} & =\left(\hat{\mathbf{v}}_{s} \cdot \hat{\mathbf{k}}_{i}\right)\left(\hat{\mathbf{h}}_{i} \cdot \hat{\mathbf{k}}_{s}\right) R_{h}-\left(\hat{\mathbf{h}}_{s} \cdot \hat{\mathbf{k}}_{i}\right)\left(\hat{\mathbf{v}}_{i} \cdot \hat{\mathbf{k}}_{s}\right) R_{v} \\
f_{v h} & =\left(\hat{\mathbf{h}}_{s} \cdot \hat{\mathbf{k}}_{i}\right)\left(\hat{\mathbf{v}}_{i} \cdot \hat{\mathbf{k}}_{s}\right) R_{h}-\left(\hat{\mathbf{v}}_{s} \cdot \hat{\mathbf{k}}_{i}\right)\left(\hat{\mathbf{h}}_{i} \cdot \hat{\mathbf{k}}_{s}\right) R_{v}
\end{aligned}
$$




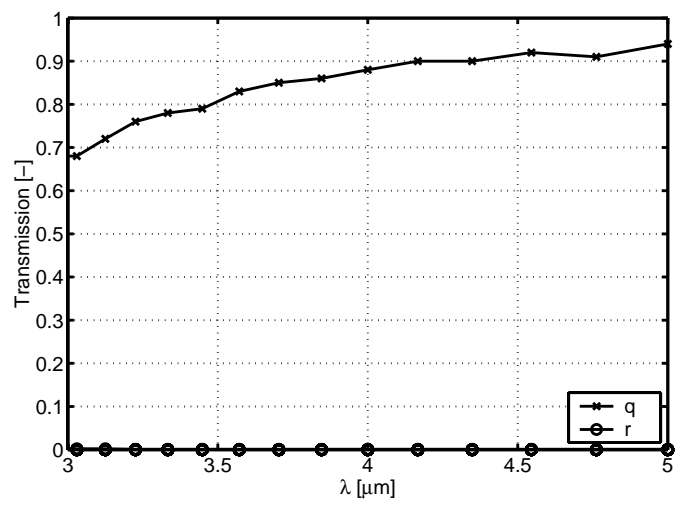

(a)

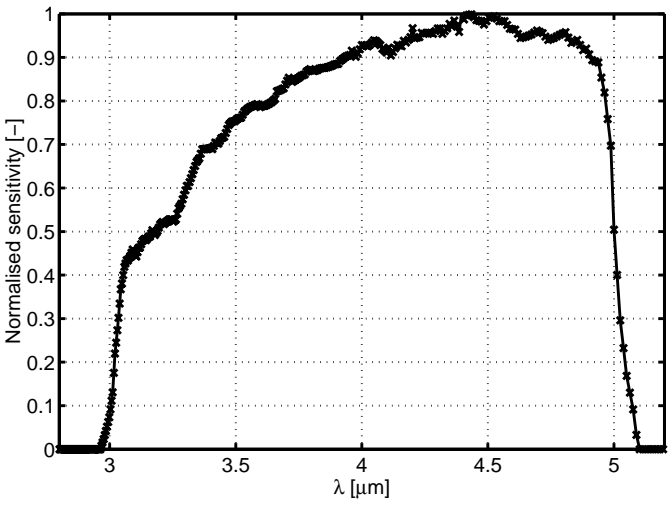

(b)

Figure 1. (a) The transmission factors $q$ and $r$ of a TIR polarisation wire grid filter (ZnSeAr/Au) as function of the wavelength $\lambda$ as specified by the manufacturer Specac. (b) The normalised spectral sensitivity of the Radiance HS.

In these expressions $\hat{\mathbf{v}}$ and $\hat{\mathbf{h}}$ are unit vectors in the vertical and horizontal directions respectively with respect to the plane of incidence.

\section{MEASUREMENT SETUP}

\subsection{Polarimateric infrared camera setup}

The polarimetric infrared camera setup consist of a Radiance HS mid wave infrared (MWIR) camera $(\lambda=3$ to $5 \mu \mathrm{m})$ and a rotating polarisation wire grid polariser. ${ }^{2,3}$ The linear polarisation filter is represented by the Mueller matrix $M_{p f} 9$ :

$M_{p f}(\phi, q, r)=\frac{1}{2}\left(\begin{array}{cccc}q+r & (q-r) \cos 2 \phi & (q-r) \sin 2 \phi & 0 \\ (q-r) \cos 2 \phi & (q+r) \cos ^{2} 2 \phi+2 \sqrt{q r} \sin ^{2} 2 \phi & (q+r-2 \sqrt{q r}) \cos 2 \phi \sin 2 \phi & 0 \\ (q-r) \sin 2 \phi & (q+r-2 \sqrt{q r}) \cos 2 \phi \sin 2 \phi & (q+r) \sin ^{2} 2 \phi+2 \sqrt{q r} \cos ^{2} 2 \phi & 0 \\ 0 & 0 & 0 & 2 \sqrt{q r}\end{array}\right)$,

with $\phi$ the orientation of the filter ( $\phi=0^{\circ}$ is horizontal), $q$ and $r$ the transmission factors of the polarisation filter. An ideal filter has a unity transmission factor for $q$ and zero transmission for $r$.

The transmission factors of the wire grid polarisation filter as function of wavelength $\lambda$ are given in Figure 1(a). The transmission factor $u$ for this specific filter is very close to zero (it is less than $0.5 \%$ over the wavelength band). That means that it almost totally blocks the radiation that is perpendicular to the transmission axes of the polarisation filter. However, the transmission factor $q$ does not reach unity and it even exhibits some variation over the wavelength. The average value is around $85 \%$, which means that on average $15 \%$ is absorbed or reflected by the filter. Besides the transmission through the filter, the spectral sensitivity of the camera is given in Figure 1(b). The spectral response has been measured using a filter wheel spectral emitter ${ }^{10}$ and is normalised to the maximum spectral sensitivity. The spectral transmission of the polarisation filter is more constant than the spectral sensitivity of the IR camera.

Using the Mueller matrix of the polarisation filter in Equation 20 the resulting Stokes vector of an arbitrary input Stokes vector $S=[I Q U V]^{T}$ can be determined. This is performed by multiplying the matrix and the vector. This new Stokes vector $S_{L P}$ is measured by an infrared camera. Normally infrared cameras are not polarisation sensitive, so only the first element of the Stokes vector (the total intensity) is to be used. The total intensity $I_{L P}$ of an arbitrary Stokes vector measured behind a linear polarisation filter with orientation $\phi$ is given by:

$$
\begin{aligned}
S_{L P}(\phi) & =M_{p f}(\phi, q, r) \cdot S \\
I_{L P} & =S_{L P}(1)=\frac{1}{2}[(q+r) I+(q-r) Q \cos 2 \phi+(q-r) U \sin 2 \phi] .
\end{aligned}
$$




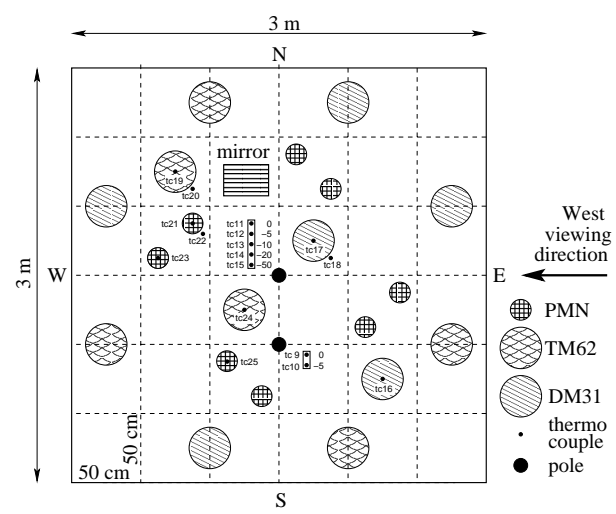

(a)

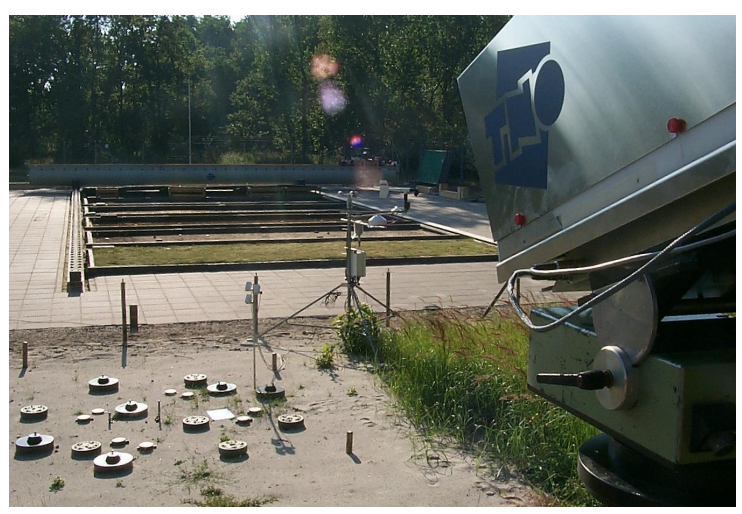

(b)

Figure 2. The layout of the test field: schematically in (a) and an overview photo in (b). The thermocouples indicated by tc\# that are drawn in the middle of the landmine are attached to the top of the landmine, while the thermocouples drawn on the lower right side of the landmine are attached to the bottom. The thermocouples indicated by dots in a box measure the temperature of the sand at different depths. The mirror in the test field has been used to measure the specular sky radiance.

If the transmission factor $r$ is assumed to be zero (which is a reasonable assumption for the polarisation filter as shown in Fig. 1) then $I_{L P}$ reduces to

$$
I_{L P}(\phi)=\frac{1}{2} q[I+Q \cos 2 \phi+U \sin 2 \phi] .
$$

For a unity value of $q$ this equation is known as Maulus's law. ${ }^{11}$ It is clear that by using our two-point calibration procedure, ${ }^{2}$ the average of the transmission factor $q$ is corrected for. By doing measurements at different angles the values for $I, Q$ and $U$ can be solved from this equation. Obviously circular polarisation $(V)$ cannot be solved from Equation 23, since the equation does not depend on $V$.

\subsection{Test field layout}

An overview of the layout of the testfield as seen from the polarimetric IR camera point of view is shown in Fig. 2. The test field contains three different types of surrogate landmines: a rubber top PMN, a plastic TM62 and a metal DM31. These surrogate landmines are laid on top of bare sand. Attached to these landmines and in the soil are thermocouples. The measured temperatures are used to estimate the blackbody radiance of the mines and the background.

The polarimetric camera is placed at a height of $2.88 \mathrm{~m}$ and is looking downwards at a nadir (off-normal) angle of $70^{\circ}$. With the $25 \mathrm{~mm}$ lens, the camera has a field of view of $18^{\circ}$ by $18^{\circ}$.

In the field of view there is also a mirror present. This mirror is placed horizontally and reflects the sky radiance for the specular direction. This sky radiance is a necessary input for the specular model.

Besides the mirror, the test facility is also equipped with a small meteorological station. This station measures the short wave irradiation (in $\left[\mathrm{W} \mathrm{m}^{-2}\right]$ ). This sensor is used to estimate the solar radiation (which falls mostly within the sensor's wavelength band).

\section{MEASUREMENTS}

For validation of the models, measurements were performed on 16 July 2002. The camera was oriented Westward and measurements were taken around sun set at 3 minutes interval (between 16:30 and 22:30). At this day in summer at 18:30, the position of the sun has an elevation of $20^{\circ}$ when it is exactly in the West $\left(270^{\circ}\right.$ azimuth). Since the IR polarimetric setup is looking West and downwards under an angle of $20^{\circ}$, this means that the sun is exactly in the specular path.

The images in Figure 3 give an overview of the influence of the orientation of the sun with respect to the scene. At 16:30 the sun is still more than $20^{\circ}$ from the specular path in azimuth. All the plastic landmines have a negative $Q$-parameter. At 18:30 the sun is almost exactly in the specular path. The $Q$-parameters of all the landmines have changed sign and are now positive. Also the landmines have a higher intensity; this is particularly visible on the metal DM31s. The $U$-parameters exhibit an interesting behaviour. All the landmines on the left of the centre have a positive $U$-parameter, whereas the 
landmines on the right have a negative $U$-parameter. The asymmetry of the $U$ parameter is expected for these horizontal surfaces because of the definition of $U$ and the camera's vertical alignment with the mine surface normals. In the centre of the image, the mine surfaces have no apparent left-right tilt and, hence, $U$ vanishes. As we look left or right, the surface has an apparent tilt, which affects $U$. Finally the three Stokes parameters are shown at 20:30, when the sun has set behind the trees. In both the $I$ and $Q$-parameter, more detail is available in the data, but due to the uniform scaling of this figure this is not visible in the printed figure.

\section{MODEL VALIDATION AND PARAMETER ESTIMATION}

\subsection{Model inputs}

For the two models three inputs are necessary. The specular model needs the sky radiance and the blackbody radiance as input. The BRDF model only needs the solar radiance as input.

\subsubsection{Sky radiance}

The radiance from the mirror as measured by the polarimetric IR camera $I_{m i}$ is given by:

$$
I_{m i}=\rho_{m i} I_{s k y}+\left(1-\rho_{m i}\right) I_{B B}\left(T_{m i}\right),
$$

with $\rho_{m i}$ the reflection coefficient of the mirror, $I_{s k y}$ the real sky radiance and $T_{m i}$ the surface temperature of the mirror. The reflection coefficient is unknown but it is expected to be higher than $95 \%$. By assuming a unity reflection coefficient (i.e. $I_{s k y}=I_{m i}$ ) only a small error is made.

\subsubsection{Blackbody radiance}

Thermocouples measure the surface and other temperatures of the surrogate landmines. Equation 3 is used to estimate the blackbody radiance for these measured temperatures. The accuracy of the thermocouples is in the order of $0.1 \mathrm{~K}$.

The disadvantage of the thermocouples is that they may not measure the exact surface temperature of the landmines, since they are glued to the surface. Moreover, the thermocouples in the sand are covered by a small layer of sand. Finally not all landmines in this setup are equipped with a thermocouple. However, for a given refractive index of the specular model and the sky radiance, an estimate of the blackbody temperature is derived using Equation 1:

$$
I_{B B}\left(T_{m}\right)=\frac{I_{m}-\frac{1}{2}\left[\rho_{s}\left(\theta_{i}\right)+\rho_{p}\left(\theta_{i}\right)\right] I_{s k y}}{1-\frac{1}{2}\left[\rho_{s}\left(\theta_{i}\right)+\rho_{p}\left(\theta_{i}\right)\right]} .
$$

This estimate can be subsequently used to estimate the Stokes element $Q$.

\subsubsection{Solar radiance}

The spectral sensitivity of the short wave sensor of the meteorological station is wider than the MWIR camera, so this measurement cannot be used directly as a measure for the solar irradiation as input for the BRDF model. Instead it is used to make an estimation of the average transmission: $\tau_{\text {sun }}$ :

$$
\tau_{\text {sun }}=I_{\text {irr }} / I_{\text {irr }, \max }
$$

with $I_{i r r}$ the measured irradiation and $I_{i r r, \max }=1366.1 \mathrm{Wm}^{-2}$ the solar constant. Consequently the radiance of the sun $I_{\text {sun }}$ is given by:

$$
I_{\text {sun }}=I_{B B}\left(T_{\text {sun }}\right) \quad \tau_{\text {sun }},
$$

with $T_{\text {sun }}=5779 \mathrm{~K}$ the surface temperature of the sun.

The orientation of the sun is fully determined by the time of the measurements. Herein, it is assumed that the refraction through the atmosphere is negligible. 


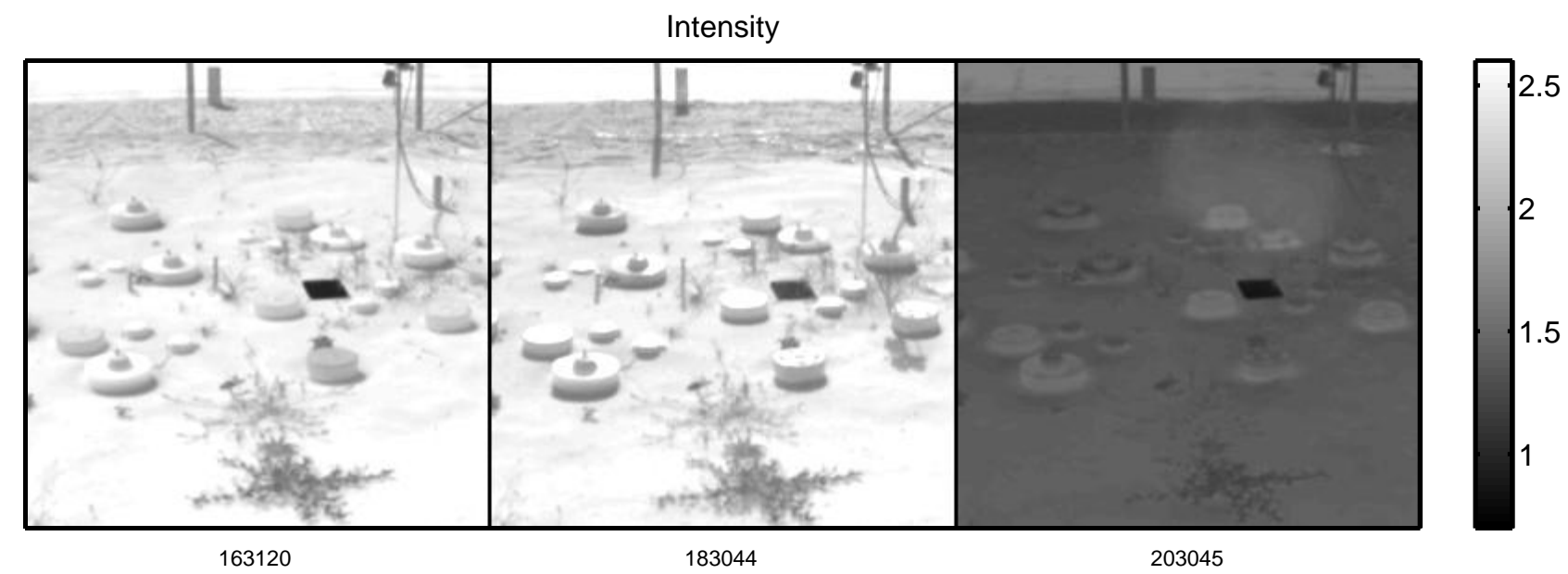

(a)

\section{Q-Component}

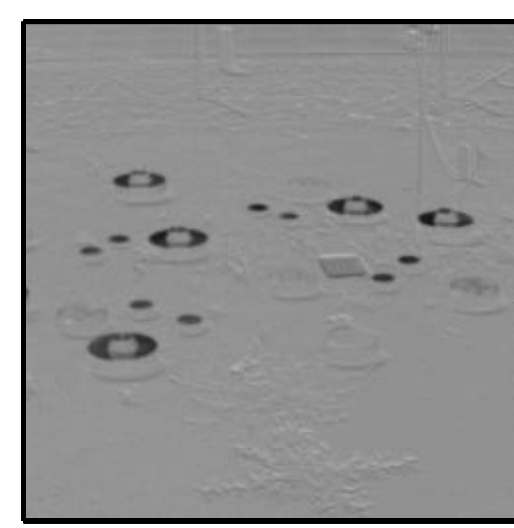

163120

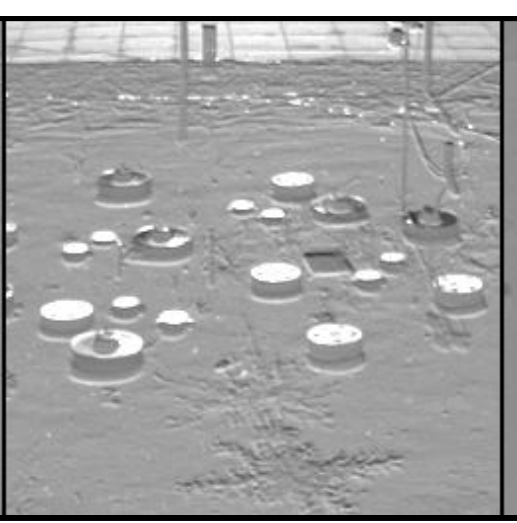

183044

(b)

U-Component

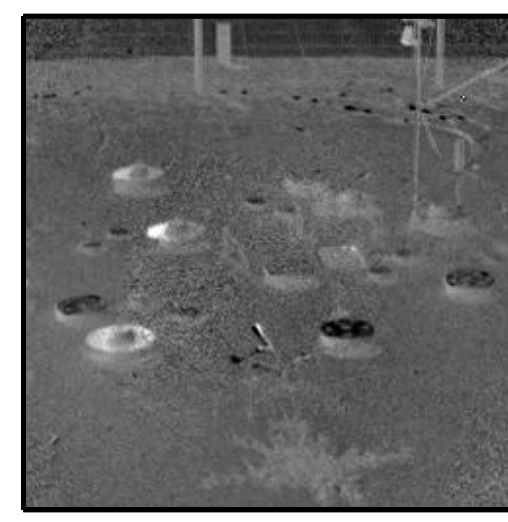

163120

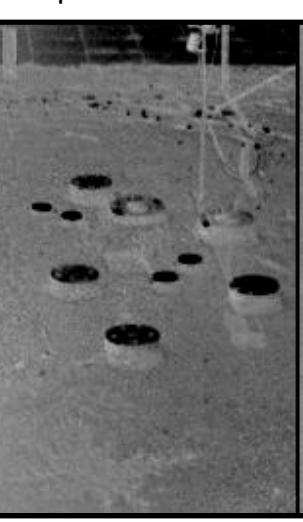

183044

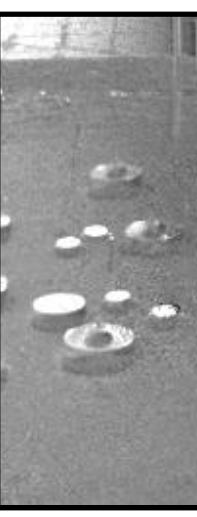

(c)

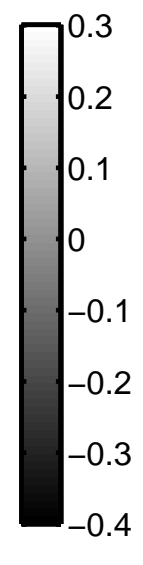

203045

) 


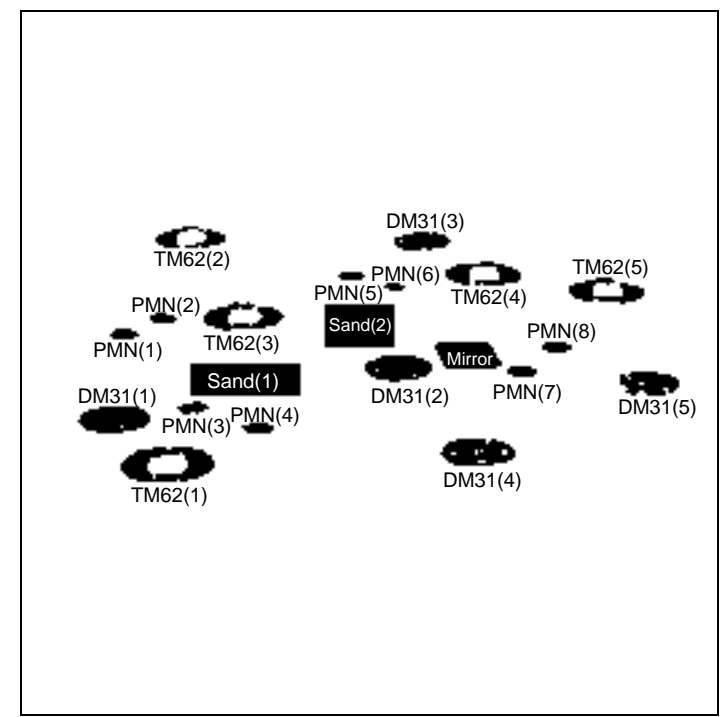

Figure 4. The definition of the different objects.

\subsection{Parameter estimation}

The specular and the BRDF model contain the following parameters: the refractive indices $\left(n_{1}\right.$ and $\left.n_{2}\right)$ and the surface roughness $(\sigma)$. For a given set of model parameters and model inputs an estimate of the Stokes vector at time $t$ is calculated: $I_{\text {model }}(t), Q_{\text {model }}(t)$ and $U_{\text {model }}(t)$. The orientation of the sun is uniquely defined by the time.

Measurements as described in Section 4 are performed as functions of time. The Stokes vectors are averaged over the different objects. The definition of the different objects is given in Figure 4. For each object and each time $t$ the average Stokes vector is calculated. The Stokes vector for object $i$ consist of the elements $I_{m}^{i}(t), Q_{m}^{i}(t)$ and $U_{m}^{i}(t)$.

A measure for the fit between the estimated model values $I_{e}, Q_{e}$ and $U_{e}$ (with given parameters) and the measured Stokes vector values is given by the sum square error (SSE), which is defined for object $i$ as:

$$
\begin{aligned}
S S E_{i}\left(n_{1}, n_{2}, \sigma\right)= & \sum_{j=1}^{N}\left(I_{m}^{i}\left(t_{j}\right)-I_{e}\left(t_{j}, n_{1}, n_{2}, \sigma\right)\right)^{2}+\sum_{j=1}^{N}\left(Q_{m}^{i}\left(t_{j}\right)-Q_{e}\left(t_{j}, n_{1}, n_{2}, \sigma\right)\right)^{2} \\
& +\sum_{j=1}^{N}\left(U_{m}^{i}\left(t_{j}\right)-U_{e}\left(t_{j}, n_{1}, n_{2}, \sigma\right)\right)^{2}
\end{aligned}
$$

with $N$ is the number of measurements (222 in this case) and $t_{j}$ the time that each single measurement is performed. By minimising the $S S E$ the optimal model parameters for each object are determined. This optimisation process is performed using the Nelder-Mead minimalisation procedure. ${ }^{12}$

\subsection{Results}

Using the model inputs as described in Section 5.1 and the parameter estimation procedure in Section 5.2, the parameters for every object can be determined. In Figure 5 a comparison between the specular model and the combined model is shown for one PMN surrogate landmine. For this PMN landmine the sun is in the specular path at an azimuth of around $267^{\circ}$.

The specular model misses completely the measured increase in the Stokes intensity parameter $I$. This increase is due to the solar reflection. At $267^{\circ}$ the measured intensity is around $2 \mathrm{Wm}^{-2} \mathrm{sr}^{-1}$ higher than predicted. Outside this solar peak the agreement is reasonable though the prediction is always higher than the measurements. An explanation for this overestimated intensity (while the mine is cooling down) is that the thermocouple is fixed somewhat inside the top of the PMN and since the landmine is slowly cooling down the actual surface temperature may be less than the measured temperature inside the mine. 


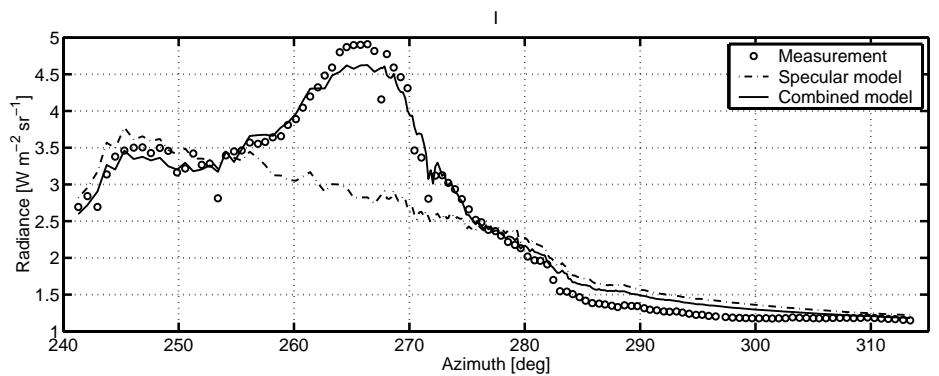

(a)

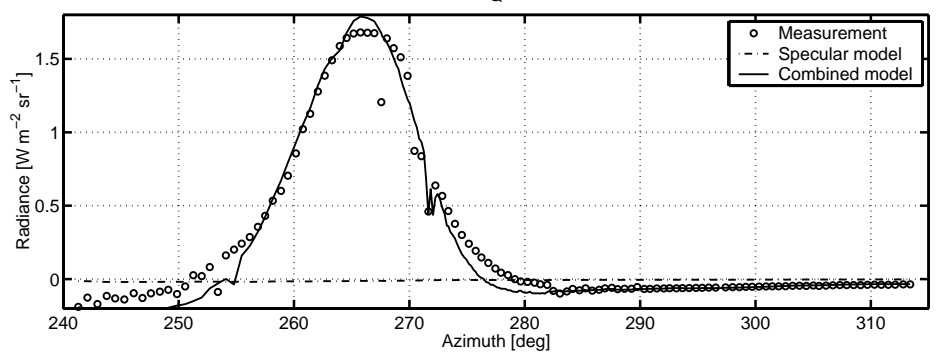

(b)

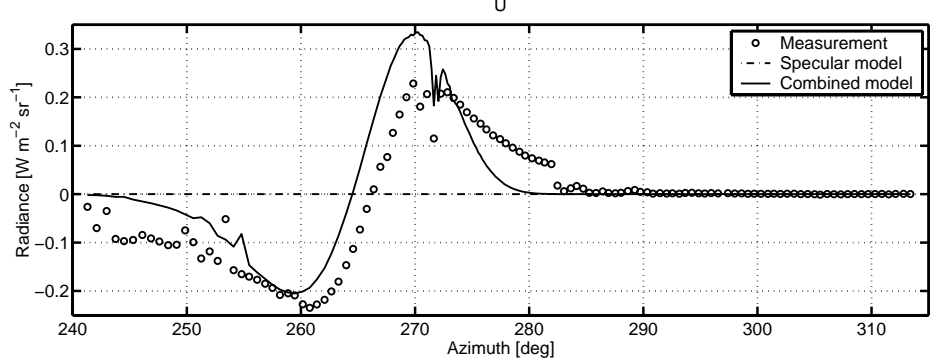

(c)

Figure 5. The $I, Q, U$ as function of the azimuth of the sun. Two models are used here to approximate the measured values. One model is the specular model, which has as inputs the thermocouple data and the sky radiance as reflected by the mirror. The other model is the combined model which has the solar radiation and orientation as additional input.

The difference between the measurement of the Stokes parameter $Q$ and the specular model is even larger. The specular model predicts a negative value of $Q$ for all azimuth angles. However, due to solar reflection the $Q$ parameter becomes positive and even reaches to $1.7 \mathrm{Wm}^{-2} \mathrm{sr}^{-1}$.

For the Stokes parameter $U$ the specular model predicts a zero value for all azimuth angles, since there are no diagonally oriented surfaces modelled. However, the measurement first shows a negative value for $U$, followed by a positive value for $U$. This effect is also observed in Figure 3(c).

The combined model, on the other hand, shows a much better agreement between the measured and modelled values for $I$ and $Q$. However a few differences remain. For the Stokes vector $I$ it does not fully reach up to the measured peak for azimuth values around $267^{\circ}$ (only to $90 \%$ of the peak). Also for the combined model there is an over estimation of the intensity for azimuth angles larger than $280^{\circ}$.

The combined model gives a reasonable prediction for the Stokes parameter $Q$. It has a small overshoot (5\%) and the width of the peak seems to be smaller than the measurements.

Finally the predicted shape of the Stokes vector $U$ compares reasonably to the measurements. However, there seems to be an offset in azimuth of around $3^{\circ}$. Furthermore, the prediction before $260^{\circ}$ and after $275^{\circ}$ is closer to zero than the measurements. This suggests that the solar response should be widened (i.e. that the surface is more rough), but the shape in these regions does not seem to match the measurements. This mismatch as well as the mismatch in peak fitting in $I$ and 


\begin{tabular}{|c|c|c|c|c|c|c|c|c|c|c|c|c|c|c|}
\hline \multirow[b]{3}{*}{ object } & \multicolumn{7}{|c|}{ No thermocouple } & \multicolumn{7}{|c|}{ Thermocouple } \\
\hline & \multicolumn{2}{|c|}{ Specular } & \multicolumn{4}{|c|}{ Combined } & \multirow[b]{2}{*}{ avg } & \multicolumn{2}{|c|}{ Specular } & \multicolumn{4}{|c|}{ Combined } & \multirow[b]{2}{*}{ avg } \\
\hline & $n_{2}$ & RMS & $n_{1}$ & $\sigma$ & $n_{2}$ & RMS & & $n_{2}$ & RMS & $n_{1}$ & $\sigma$ & $n_{2}$ & RMS & \\
\hline DM31(1) & 1.009 & 0.163 & 1.761 & 0.151 & 1.086 & 0.030 & + & 0.999 & 0.235 & 1.793 & 0.139 & 1.491 & 0.113 & + \\
\hline DM31(2) & 1.009 & 0.181 & 1.357 & 0.096 & 1.009 & 0.029 & + & 1.008 & 0.300 & 1.325 & 0.088 & 1.330 & 0.099 & + \\
\hline DM31(3) & 1.009 & 0.167 & 1.331 & 0.096 & 1.009 & 0.027 & + & 1.010 & 0.282 & 1.305 & 0.088 & 1.296 & 0.090 & + \\
\hline DM31(4) & 1.009 & 0.170 & 1.507 & 0.139 & 1.009 & 0.032 & + & 1.159 & 0.257 & 1.407 & 0.136 & 1.523 & 0.176 & - \\
\hline DM31(5) & 1.009 & 0.185 & 1.193 & 0.050 & 1.310 & 0.035 & - & 1.075 & 0.294 & 1.147 & 0.047 & 1.349 & 0.139 & + \\
\hline average & & & 1.489 & 0.120 & 1.028 & & & & & 1.393 & 0.090 & 1.367 & & \\
\hline PMN(1) & 1.009 & 0.271 & 1.909 & 0.101 & 1.135 & 0.058 & + & 1.012 & 0.370 & 1.970 & 0.094 & 1.443 & 0.129 & + \\
\hline PMN(2) & 1.009 & 0.329 & 2.051 & 0.108 & 1.193 & 0.054 & + & 1.014 & 0.490 & 2.176 & 0.105 & 1.424 & 0.113 & + \\
\hline PMN(3) & 1.009 & 0.222 & 1.892 & 0.123 & 1.198 & 0.052 & + & 1.014 & 0.317 & 2.028 & 0.124 & 1.432 & 0.095 & + \\
\hline PMN(4) & 1.009 & 0.417 & 2.007 & 0.097 & 1.138 & 0.054 & + & 1.015 & 0.661 & 2.086 & 0.090 & 1.311 & 0.128 & + \\
\hline PMN(5) & 1.009 & 0.362 & 1.677 & 0.094 & 1.246 & 0.065 & + & 1.014 & 0.530 & 1.707 & 0.097 & 1.475 & 0.125 & + \\
\hline PMN(6) & 1.009 & 0.434 & 1.853 & 0.102 & 1.130 & 0.076 & 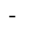 & 1.014 & 0.628 & 1.921 & 0.106 & 1.415 & 0.142 & + \\
\hline $\operatorname{PMN}(7)$ & 1.009 & 0.450 & 1.583 & 0.078 & 0.999 & 0.120 & - & 1.014 & 0.729 & 1.758 & 0.092 & 1.390 & 0.139 & + \\
\hline PMN(8) & 1.009 & 0.379 & 1.580 & 0.085 & 1.339 & 0.097 & - & 1.014 & 0.759 & 1.417 & 0.061 & 1.372 & 0.197 & + \\
\hline average & & & 1.907 & 0.105 & 1.182 & & & & & 1.883 & 0.096 & 1.408 & & \\
\hline TM62(1) & 1.009 & 0.074 & 4.316 & 0.009 & 1.009 & 0.074 & - & 1.341 & 0.131 & 1.351 & 0.052 & 1.501 & 0.096 & + \\
\hline TM62(2) & 1.323 & 0.025 & 4.132 & 0.010 & 1.323 & 0.025 & - & 1.824 & 0.177 & 3.331 & 0.021 & 1.869 & 0.176 & - \\
\hline TM62(3) & 1.248 & 0.040 & 1.150 & 0.081 & 1.409 & 0.011 & + & 1.642 & 0.153 & 3.148 & 0.022 & 1.715 & 0.148 & - \\
\hline TM62(4) & 1.009 & 0.185 & 1.193 & 0.050 & 1.310 & 0.035 & + & 1.270 & 0.285 & 4.156 & 0.010 & 1.270 & 0.285 & - \\
\hline TM62(5) & 1.009 & 0.103 & 1.118 & 0.058 & 1.373 & 0.025 & + & 1.503 & 0.189 & 3.945 & 0.009 & 1.503 & 0.189 & - \\
\hline average & & & 1.154 & 0.063 & 1.364 & & & & & 1.351 & 0.052 & 1.501 & & \\
\hline sand(1) & 1.009 & 0.020 & 1.295 & 0.337 & 1.009 & 0.005 & + & 1.166 & 0.201 & 2.832 & 0.278 & 1.742 & 0.152 & - \\
\hline sand(2) & 1.009 & 0.019 & 1.284 & 0.339 & 1.009 & 0.005 & + & 1.257 & 0.186 & 2.204 & 0.238 & 1.725 & 0.148 & - \\
\hline average & & & 1.289 & 0.338 & 1.009 & & & & & & & & & \\
\hline
\end{tabular}

Table 1. Estimates of the refractive indices ( $n_{1}$ is from the BRDF model, $n_{2}$ is from the specular model), the surface roughness $\sigma$ and the sum square error (SSE). Estimates have been made using the thermocouples and estimates for the blackbody radiance.

$Q$ is some indication that the BRDF model used here does not fully model the behaviour. However, the combined model gives a large improvement over the specular model and the predictions are still usable.

For the rest of the landmines and the sand background the model parameters and the root mean square (RMS) error is given in Table 1. The RMS error of both the specular model and the combined model is lower for the calculated blackbody radiance (i.e. without a thermocouple) than for the models that use the thermocouple for estimating the blackbody radiance.

Furthermore the error is almost always significantly lower for the combined model than for the specular model. The only exceptions are for most of the TM62's, where the combined model error is equal to the specular model. This dummy TM62 is painted and has a very smooth and shiny surface in the visible wavelengths. It is expected that for the MWIR wavelength band the surface is also very smooth as the optimal $\sigma$ is very low compared to other surfaces. Consequently the measurements are very sensitive to the exact orientation of the landmine, i.e. a small tilt has a large effect on whether or not the sun is in the specular path. Problems may also arise due to strong subsurface diffuse scattering in the paint, which is not correctly modelled by setting the diffuse scattering to zero.

The other surfaces of the DM31, PMN and the sand background are better modelled using the combined model than the specular model (since the RMS error is lower). The DM31 and the PMN have comparable surface roughnesses of 0.120 and 0.105 respectively, whereas the sand background has a much higher surface roughness of 0.338 . The refractive indices of the sand are the lowest, followed by the DM31, whereas the refractive indices for the PMN are the highest.

Since the sand has the lowest refractive indices and the highest surface roughness the polarisation contrast is the lowest. The DM31 mimics this behaviour by having a somewhat large refractive index and a smaller surface roughness. Therefore the DM31 is more difficult to distinguish from the background. Finally the PMN has the highest refractive indices and the smallest surface roughnesses and therefore it is more easily detectable using polarimetric features.

\section{CONCLUSIONS AND DISCUSSION}

The detection of landmines using normal infrared cameras that only measure intensity is limited by the presence of clutter (stones, soil variations). Using a polarimetric infrared camera permits not only the measurement of intensity but also of two additional polarimetric signals. Our specular model accurately predicts the polarimetric signal for landmines under conditions where the sun is of limited influence. If the sun is close to the specular path then the predictions of the specular 
model are no longer valid, as is shown in this paper. The polarimetric contrast in $Q$ may become zero or may even turn from negative into positive. Furthermore the $U$ value does not remain zero as predicted but may have a significant amplitude.

The specular model also does not give insight in the modelling of the soil background. Since the refractive index is the only variable parameter, it estimates that the soil background has a very low (almost unity) refractive index to explain the measurement results.

By enhancing the specular model with a BRDF model (that has been used for an image simulator previously) a new combined model is created. The influence of point sources like sunlight is modelled using an approximation to the surface BRDF, while the specular model includes the thermal emission and an approximation to scattered skylight.

This combined model functions well for the DM31 and the PMN (dummy) landmines and the sand background. For the TM62 dummy which has a smooth paint finish the combined model predictions are erratic. It is expected that small variations in placement of these specific landmines has a large effect on the signal, since the optimal fit between the model and measurement indicates that the surface roughness is very low.

The two other landmines have a larger roughness, but they differ in the refractive indices. The DM31 has a lower refractive index and therefore its polarimetric signal is lower than the PMN.

Finally the sand background has both the lowest refractive index as well as the highest surface roughness. These values explain why the polarimetric signal of the background is very low compared to the landmines. As a consequence the landmines are distinguishable from the background even when the intensity (or blackbody temperature) is the same.

\section{ACKNOWLEDGEMENTS}

The work presented in this paper is sponsored by the Netherlands Ministry of Defense.

The efforts of BAB and WJL were supported in part by the US Army, Night Vision Electronic Systems Directorate.

\section{REFERENCES}

1. J. G. M. Schavemaker, E. den Breejen, K. W. Benoist, K. Schutte, P. Tettelaar, M. de Bijl, and P. J. Fritz. LOTUS field demonstration of integrated multisensor mine-detection system in Bosnia. In R. S. Harmon, J. T. Broach, and J. John H. Holloway, editors, Proc. SPIE Vol. 5089, Detection and Remediation Technologies for Mines and Minelike Targets VIII, Orlando (FL), USA, Apr. 2003.

2. F. Cremer, W. de Jong, and K. Schutte. Infrared polarisation measurements and modelling applied to surface laid anti-personnel landmines. Optical Engineering, 41(5):1021-1032, May 2002.

3. F. Cremer, W. de Jong, K. Schutte, J. T. Johnson, and B. A. Baertlein. Surface mine signature modeling for passive polarimetric IR. In J. T. Broach, R. S. Harmon, and G. J. Dobeck, editors, Proc. SPIE Vol. 4742, Detection and Remediation Technologies for Mines and Minelike Targets VII, pages 51-62, Orlando (FL), USA, Apr. 2002.

4. B. A. Baertlein and J. T. Johnson. Physically based simulation of passive polarimetric IR mine signatures. In J. T. Broach, R. S. Harmon, and G. J. Dobeck, editors, Proc. SPIE Vol. 4742, Detection and Remediation Technologies for Mines and Minelike Targets VII, pages 31-42, Orlando (FL), USA, Apr. 2002.

5. P. Beckmann and A. Spizzichino. The scattering of electromagnetic waves from rough surfaces. Pergamon, New York, NY, 1963.

6. K. E. Torrance and E. M. Sparrow. Theory for off specular reflection from roughened surfaces. J. Opt. Soc. Am. (JOSA), (9):1105-1114, Sept. 1967.

7. J. R. Maxwell, J. Beard, S. Weiner, and D. Ladd. Bidirectional reflectance model validation and utilization. Technical Report AFAL-TR-73-303. Enviromental Research Institute of Michigan (ERIM), Ann Arbor, MI, 1973.

8. L. Tsang, J. A. Kong, and R. T. Shin. Theory of Microwave Remote Sensing. Wiley, New York, NY, 1985.

9. M. H. Smith, M. A. Miller, R. V. Blumer, M. A. Stevens, D. M. Teale, and J. D. Howe. Infrared Stokes polarimeter calibration. In D. H. Goldstein, D. B. Chenault, W. G. Egan, and M. J. Duggin, editors, Proc. SPIE Vol. 4133, Polarization Analysis, Measurement, and Remote Sensing III, pages 55-64, San Diego (CA), USA, July 2000.

10. A. N. de Jong, R. A. W. Kemp, and J. Winkel. Description of 2 filter wheel spectral emitters (FWSE). Technical Report FEL-98-I070, TNO Physics and Electronics Laboratory, The Hague, The Netherlands, Apr. 1998.

11. E. Hecht. Optics. Addison-Wesley publishing company, Reading (MA), USA, second edition, 1987.

12. J. C. Lagarias, J. A. Reeds, M. H. Wright, and P. E. Wright. Convergence properties of the nelder-mead simplex method in low dimensions. SIAM Journal of Optimization, 9(1):112-147, 1998. 